\title{
Pulsed electric field in combination with vacuum impregnation with trehalose improves the freezing tolerance of spinach leaves
}

\author{
Pui Yeu Phoon ${ }^{\mathrm{a}, 1}$, Federico Gómez Galindo ${ }^{\mathrm{b}, *}$, António Vicente ${ }^{\mathrm{b}}$, Petr Dejmek ${ }^{\mathrm{a}}$ \\ ${ }^{a}$ Department of Food Technology, Engineering and Nutrition, Lund University, P.O. Box 124, SE-221 00 Lund, Sweden \\ ${ }^{\mathrm{b}}$ IBB-Institute for Biotechnology and Bioengineering, Centro de Engenharia Biológica, Universidade do Minho, \\ Campus de Gualtar, 4710-057 Braga, Portugal
}

Received 1 October 2007; received in revised form 3 December 2007; accepted 9 December 2007

Available online 31 December 2007

\begin{abstract}
Pulsed electric fields in combination with vacuum infusion have been utilized to impregnate cells with trehalose, aiming at substantially improving the freezing tolerance of spinach leaves. Spinach samples were first treated with ten trains of bi-polar, rectangular electric field pulses with a nominal electric field strength of $580 \mathrm{~V} / \mathrm{cm}$ and immediately immersed in a $40 \%$ (w/w) solution of trehalose under vacuum for $20 \mathrm{~min}$. The samples were kept in the trehalose solution for $2.5 \mathrm{~h}$ at atmospheric pressure, immersed in deionised water at $4{ }^{\circ} \mathrm{C}$ overnight, frozen in liquid nitrogen and thawed in water at room temperature. The leaves were evaluated for cell damage with microscopic observations and wilting tests. The results provided evidence that the impregnation with trehalose by the combined actions of electric fields and vacuum impregnation drastically improved the freezing tolerance of the spinach leaves.
\end{abstract}

(c) 2008 Published by Elsevier Ltd.

Keywords: Pulsed electric fields; Vacuum impregnation; Cryoprotection; Cold stress

\section{Introduction}

Freezing is an excellent and fairly widespread method for preserving food products, including fruits and vegetables, providing a high stability to health-beneficial micronutrients such as folates (Phillips et al., 2005). However, the freezing of vegetables, for example spinach, may alter quality characteristics such as flavour and texture, which, in turn, could affect its marketing potential.

In a typical industrial process, vegetables such as spinach are usually frozen quickly so as to produce small ice crystals. However, these crystals may grow larger over time through recrystallization. Recrystallization in frozen foods can result in membrane damage, a reduced water-holding

\footnotetext{
${ }^{*}$ Corresponding author.

E-mail addresses: federico.gomez@deb.uminho.pt, federico.gomez@ food.lth.se (F.G. Galindo).

${ }^{1}$ Present address: Department of Food Science, Purdue University, USA.
}

capacity (high drip loss), loss of nutrients (Breton et al., 2000), lipid oxidation and the formation of off-flavours (Stanley, 1991).

Cryoprotection of horticultural products has been a matter of concern for the food industry in its search for efficient ways of improving the quality of frozen-thawed products (Gómez Galindo and Sjöholm, 2004). Research on the cryoprotection of plant tissues has made considerable progress through a better understanding of the natural protective mechanisms of plant tissues during winter survival. The accumulation of osmotically active substances, also called compatible solutes (COS), in the cytosol of the cells is part of this protective mechanism. COS are synthesized by many organisms ranging from bacteria to animals and plants in response to desiccation, osmotic stress, salt stress or low temperature (Taiz and Zeiger, 2002). This chemically heterogeneous group of substances comprises some amino acids (e.g. proline), quaternary ammonium compounds (e.g. betaine), numerous sugars (e.g. sucrose and trehalose) and several other substances (see Yancey, 2005 for a review). 
The protective role of sugars has been widely studied. There is a wealth of information on their stabilizing effects on biological molecules, cells and organisms, which indicates their functional role in the stress tolerance of many, although not all, organisms (Hincha et al., 2006). Apart from decreasing the chemical potential of water and the freezing point in the cytosol, the hydrophilic nature of sugars is well-suited for stabilizing the cell membrane through hydrogen bonding between the hydroxyl groups on the sugar and the polar residues in phospholipids. This prevents dehydration effects in membranes (Danyuluk et al., 1998).

Attempts have been made to improve the resistance of horticultural products to freezing damage by treating the tissues with cryoprotectants. Industrial techniques, such a vacuum impregnation (VI), have been used to promote compositional changes on porous tissues (e.g. apples), by filling porous fractions of the product with an external solution of the cryoprotectant. Resistance to freezing damage has then been improved through the reduction of freezable water (Martínez-Monzó et al., 1998). However, when applying VI, the solution containing the cryoprotectant is located in the apoplast, whereas in nature, cryoprotectants accumulate inside the cell conferring their protective effects to the plant tissues.

Sugars could be forced inside the cells through osmotic dehydration, by immersing the tissue in a hypertonic solution. To accelerate the mass transfer during this operation, pulsed electric fields (PEF) have been used to affect plasma membrane permeability properties (Ade-Omowaye et al., 2002; Taiwo et al., 2003). However, these operations will induce permanent damage on cell membrane properties and cell death (Mavroudis, 2003), consequences that will not provide cryoprotection to the tissue.

The present study reports preliminary results on the use of PEF in combination with VI to obtain a cryoprotectant solution inside the cells of spinach leaves, showing a substantial improvement of their freezing tolerance. PEF causes a transient increase in the transmembrane potential difference, modifying the plasma membrane organization and bringing it, locally, to a permeable state (Sabri et al., 1996). By strict control of the electropulsation parameters, the permeabilization may evade affecting the cell viability. Such a process can be used for transporting substances such as cryoprotectants across the cellular distance impregnating the cells.

\section{Materials and methods}

\subsection{Raw material}

Spinach (Spinacia oleracea) leaves $(8.0 \pm 0.5 \mathrm{~g}$ dry matter/100 g sample) were purchased in $150 \mathrm{~g}$ sealed bags at the local market (Southern Sweden). The bags were stored at $4{ }^{\circ} \mathrm{C}$ and the experiments were carried out 3-7 days before the declared expiration date of the spinach. Each time leaves were removed from the bags, these were resealed and stored cold until more leaves were required.

\subsection{Sample preparation}

Rectangular samples, $3.0 \mathrm{~cm}$ long, $0.5 \mathrm{~cm}$ wide and $0.06 \mathrm{~cm}$ thick, were cut from the spinach leaves using a sharp blade. To ensure that the thickness of each sample was even, the thickness was measured with a caliper in 2 3 different regions. All leaf samples were free from major portions of conductive tissue.

\subsection{Treatments}

\subsubsection{Electrical treatment}

The rectangular samples were placed in between two flat stainless steel electrodes ( $36 \mathrm{~mm}$ long and $9 \mathrm{~mm}$ wide) were they received electric pulses at room temperature. The sandwich structure of electrodes and sample was wrapped with scotch tape (Scotch Magic ${ }^{\mathrm{TM}}$ ) to minimize sample evaporation. The electric pulses were delivered by a CythorLab ${ }^{\mathrm{TM}}$ electroporator (ADITUS AB, Lund, Sweden), and the parameters of the treatment were programmed by a computer software package (PulseEdit ${ }^{\mathrm{TM}}$, ADITUS AB, Lund, Sweden). Ten trains of bi-polar, rectangular electric field pulses with a nominal electric field strength of $580 \mathrm{~V} / \mathrm{cm}$ (the nominal field strength is here defined as the applied voltage divided by the separation between the electrodes, i.e. $0.06 \mathrm{~cm}$ ) were used. Each train lasted $20 \mathrm{~ms}$ and consisted in $25 \mu$ sulses. The resting period between the trains was $10 \mathrm{~s}$ to avoid heating of the samples (Fig. 1).

The CythorLab ${ }^{\mathrm{TM}}$ was programmed to measure the electrical impedance of the sample in the range $10 \mathrm{~Hz}$ to $100 \mathrm{~Hz}$ at a given time after the treatment. For these measurements, the CythorLab ${ }^{\mathrm{TM}}$ generated bi-polar current pulses with an amplitude of $\pm 1 \mathrm{~mA}$ and a wave train length of $131 \mathrm{~ms}$. The results were expressed as the change in admittance (reciprocal of the impedance, $d \mathrm{Y} \%$ ) with time, compared to pre-electroporation values.

\subsubsection{Vacuum impregnation}

The VI treatment was carried out at $20^{\circ} \mathrm{C}$ in a chamber connected to a vacuum pump. The spinach samples were immersed in a $40 \%(\mathrm{w} / \mathrm{w})$ solution of trehalose for $25 \mathrm{~min}$. This duration comprised a gradual increase of the vacuum for $3 \mathrm{~min}$, a holding time of $20 \mathrm{~min}$ at $-86 \mathrm{kPa}$ (man) and a gradual release of the vacuum for $2 \mathrm{~min}$. The VI process assured a volume fraction of trehalose in the spinach tissue of around $20 \%$. After the impregnation, the samples were kept in the trehalose solution for $2.5 \mathrm{~h}$ (relaxation time). They were then taken out and their surfaces were blotted to remove excess solution. Trehalose, being only $45 \%$ of the sucrose sweetness, is not likely to adversely influence the taste of the leaves.

\subsubsection{Combination of treatments}

Spinach samples were treated with a combination of PEF and VI prior to freezing and thawing. The various procedures and their corresponding processing steps are shown in Fig. 2, where spinach leaves were treated with 


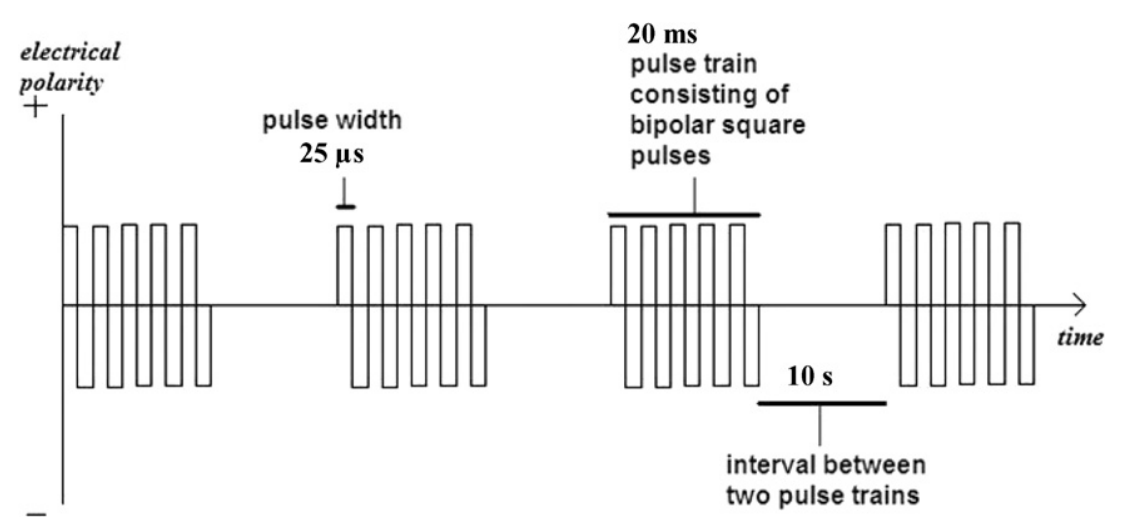

Fig. 1. A simplified diagram of the PEF treatment design. Ten trains of bi-polar, rectangular electric field pulses with a nominal electric field strength of $580 \mathrm{~V} / \mathrm{cm}$ were applied as described in the Section 2. Pulse characteristics are shown in the figure.

Process 1

$$
\mathrm{PEF} \longrightarrow \mathrm{VI} \rightarrow \text { Relaxation } \rightarrow \text { Resting } \rightarrow \text { Freezing } \rightarrow \text { Thawing }
$$

Process 2

$$
\mathrm{VI} \rightarrow \text { Relaxation } \rightarrow \text { Resting } \rightarrow \text { Freezing } \rightarrow \text { Thawing }
$$

Fig. 2. A combination of treatments for improving the freezing tolerance of spinach leaves. Each unit operation was performed as described in the Section 2.

and without PEF. Ten samples were treated with each process and the VI was applied to the leaf samples immediately after the electroporation treatment. The other processing steps were carried out as follows: the resting step was performed by submerging the samples in deionised water overnight at $4{ }^{\circ} \mathrm{C}$ to regain turgor. Freezing was done by submerging the individual samples in liquid nitrogen for $7 \mathrm{~s}$. The samples were then immediately thawed in water at room temperature.

\subsection{Analyses}

\subsubsection{Microscopic observations}

The cell integrity was evaluated by using fluorescein diacetate (FDA, Sigma-Aldrich, USA) as described by Gómez Galindo et al. (2005a) with some modifications. Leaf samples were cut into sections of $\sim 0.5 \times 0.1 \mathrm{~cm}$ using sharp blades and immediately placed in $10 \mathrm{ml}$ of deionised water. One hundred microlitres of $0.01 \%(w / v)$ FDA in acetone was added to the water, giving rise to a final FDA concentration of $2.4 \times 10^{-6} \mathrm{M}$. The sections were incubated in the FDA solution for $4.5 \mathrm{~h}$ in darkness and at room temperature. The stained sections were rinsed with deionised water and examined under fluorescent light conditions in an epi-fluorescence microscope (Olympus BX-50/BX-FLA, Japan). Undamaged cells could be easily identified by a bright fluorescence.

\subsubsection{Wilting test}

The turgidity of the treated spinach samples was evaluated by simply holding the centre of the sample with a small pincer and observing whether the leaves would bend or not.

\section{Results and discussion}

\subsection{Sample recovery after electroporation is dependent on its initial impedance}

Fig. 3 shows the effect of the pre-pulsation impedance of the spinach samples on the change in admittance (reciprocal of the impedance, $d \mathrm{Y} \%$ ) as a function of the time after electroporation. The change in electrical admittance (dY\%) was used to evaluate the progress of the cell membrane permeabilization and the recovery of the spinach after the PEF application (Angersbach et al., 2002; Bazhal et al., 2003). According to Fig. 3, where average curves from at least three measurements are displayed, this recovery was dependent on the initial impedance of the leaves. When the initial impedance of the leaves was in the range of 5000-6500 $\Omega$, the admittance displayed an increase of approximately

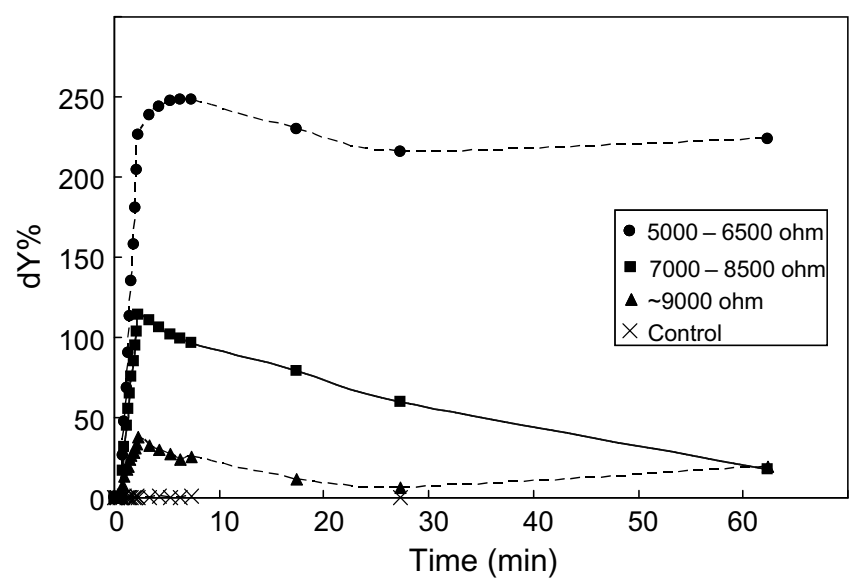

Fig. 3. The effect of the pre-pulsation impedance of spinach samples on the change of admittance (reciprocal of the impedance, $\mathrm{dY} \%$ ) as a function of the time after electroporation. Average curves of at least three replicates are shown. $\mathrm{d} Y \%$ is calculated as the difference (in percentage) between the admittance values at a certain time after pulsation and the pre-pulsation value. 
$250 \%$ of the initial value and showed no recovery $1 \mathrm{~h}$ after electroporation. For an initial impedance ranging from 7000 to $8500 \Omega$, the admittance showed an increase of around $100 \%$ of the initial value and a continuous decrease down to around $20 \% 1 \mathrm{~h}$ after PEF application. The samples were fully recovered $2.5 \mathrm{~h}$ after electroporation (not shown). When the initial impedance of the leaves was around $9000 \Omega$, the admittance increased approximately $30 \%$ and a full recovery of the cells was obtained within 25 min.

It has been demonstrated (Arora and Palta, 1991) that a partially damaged cell membrane has the ability to recover even if the damage has caused a significant ion leakage. The ATPase activity, which uses the chemical energy of ATP, is required for this recovery process, as it helps the cell to take up the leaked ions against the concentration gradient. It is a long-term physiological process and may take several hours (Arora and Palta, 1991). Our observation that the capacity of the leaves to recover after PEF treatment is apparently affected by their initial impedance might be associated with differences on the electric current flowing through the tissue during the electropulsation. A higher current might cause higher damage to the cells, affecting the recovery process. The different values of initial impedance may be a consequence of differences on cell wall structure.

\subsection{The combination of PEF and VI improves the freezing resistance of spinach leaves}

Spinach samples with initial impedance values in the range 7000-8500 $\Omega$ were used for the subsequent processing operations. The FDA and the wilting tests demonstrated that the application of "Process 1" (Fig. 2) resulted in viable, turgid spinach leaves (see Fig. 4c for a typical result). These results strongly suggested that the applied processing steps allowed the uptake of trehalose by the tissue, thus dramatically increasing the freezing resistance of the samples. The time scale for restoring the atmospheric pressure after the VI treatment corresponded to the time required for the sample to recover after the PEF treatment (Fig. 3). This suggests that the cell membranes leaked cellular contents when the vacuum was increased, as well as during most of the holding time, and that these contents were mixed with the incoming trehalose solution. The uptake of the leaked cellular contents during recovery may have facilitated the uptake of the cryoprotectant inside the cells. The active transport of trehalose has been proven in yeasts (Stambuk et al., 1996; Malluta et al., 2000) but remains to be demonstrated in leaves such as spinach. However, this idea was supported by the results from "Process 2" (Fig. 2) as the FDA and the wilting tests showed non-viable cells and totally wilted leaves (see Fig. 4d for a typical result). This was thus considered evidence that the impregnation of the trehalose in the extracellular space alone, as expected after the VI process, was insufficient for cryoprotection and that the leakage/recov- a
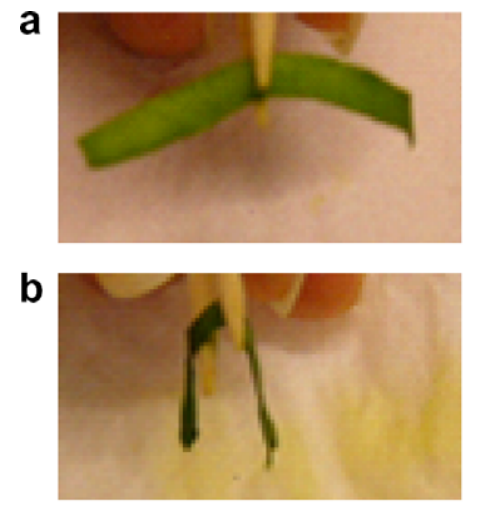

C
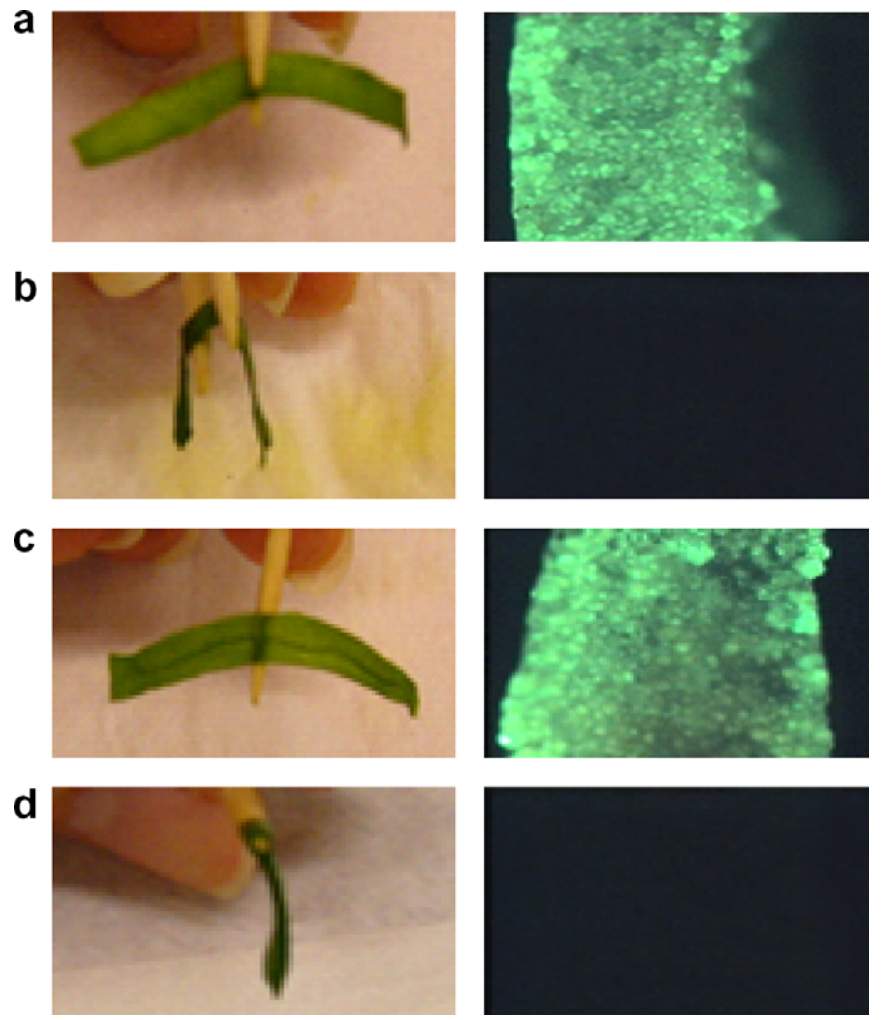

Fig. 4. The freezing tolerance of spinach leaves after different treatments. Left panel: Typical results (10 measurements) from the wilting test, performed as described in the Section 2, showing the turgidity of the samples. Right panel: Typical results (10 measurements) from microscopic observations using fluorescein diacetate to identify viable cells. Viable cells are distinguished by a bright fluorescence. (a) A fresh spinach leaf. (b) A leaf frozen in liquid nitrogen for $7 \mathrm{~s}$ and immediately thawed in water at room temperature. (c) Spinach leaves subjected to "Process 1", as described in the Section 2 and schematised in Fig. 1. (d) Spinach leaves subjected to "Process 2", as described in the Section 2 and schematised in Fig. 1.

ery process provoked by PEF was a key event providing freezing resistance to the leaves.

The freezing tolerance of any organism can never be explained by the action of merely one compound (Hincha et al., 2006). In addition to sugars, many other physiological adaptations must take place to allow an organism to survive extreme stresses such as freezing or desiccation (see Smallwood and Bowles, 2002 for a review). The capacity of spinach to undergo the complex series of metabolic events associated with the cold acclimation process has been widely reported in the literature (Somersalo and Krause, 1990; Turan et al., 2007). Moreover, these metabolic events might continue during storage if the harvesting has been done under specific climatic conditions (Gómez Galindo et al., 2005a,b). Since the samples for this study were obtained in the local market, the information concerning the pre- and post-harvest history of the spinach was insufficient. Therefore, the influence of the initial physiological status of the tissue on the freezing tolerance that was gained with trehalose by the combination of PEF and VI requires further investigation. 


\section{Concluding remarks}

The preliminary results presented herein provide evidence that the impregnating spinach leaves with the cryoprotectant trehalose with the aim of improving the freezing tolerance of spinach leaves could be achieved only when the VI process was used in combination with PEF. The combined unit operations probably allowed trehalose to be present in both the extracellular and intercellular spaces. Trehalose would then be able to protect the cell from both sides, probably in combination with possible effects of metabolic events having taken place in the leaves during pre- and post-harvest.

\section{References}

Ade-Omowaye, B.I.O., Rastogi, N.K., Angersbach, A., Knorr, D., 2002. Osmotic dehydration of bell peppers: influence of high intensity electric field pulses and elevated temperature treatment. J. Food Eng. 54, 3543.

Angersbach, A., Heinz, V., Knorr, D., 2002. Evaluation of processinduced dimensional changes in the membrane structure of biological cells using impedance measurements. Biotechnol. Prog. 18, 597-603.

Arora, R., Palta, J.P., 1991. A loss in the plasma membrane ATPase activity and its recovery coincides with incipient freeze-thaw injury and postthaw recovery in onion bulb scale tissue. Plant Physiol. 95, 846-852.

Bazhal, M., Levobka, N., Vorobiev, E., 2003. Optimisation of pulsed electric field strength for electroplasmolysis of vegetable tissues. Biosyst. Eng. 86, 339-345.

Breton, G., Danyluk, J., Ouellet, F., Sarhan, F., 2000. Biotechnological applications of plant freezing associated proteins. Biotechnol. Ann. Rev. 6, 59-101.

Danyuluk, J., Perron, A., Hounde, M., Limin, A., Fowler, B., Benhamou, N., Sarhan, F., 1998. Accumulation of an acidic dehydrin in the vicinity of the plasma membrane during cold acclimation of wheat. Plant Cell 10, 623-638.

Gómez Galindo, F., Sjöholm, I., 2004. Applying biochemical and physiological principles in the industrial freezing of vegetables: a case study on carrots. Trends Food Sci. Technol. 15, 39-43.

Gómez Galindo, F., Toledo, R.T., Sjöholm, I., 2005a. Tissue damage in heated carrot slices. Comparing mild hot water blanching and infrared heating. J. Food Eng. 67, 381-385.
Gómez Galindo, F., Elias, L., Herppich, W., Smallwood, M., Sommarin, M., Worrall, D., Gekas, V., Sjöholm, I., 2005b. On the induction of cold acclimation in carrots and its influence on storage performance. Food Res. Int. 38, 29-36.

Hincha, D.K., Zuther, E., Hundertmark, M., Heyer, A.G., 2006. The role of compatible solutes in plant freezing tolerance: a case study on raffinose. In: Chen, T.H.H., Uemura, M. (Eds.), Cold Hardiness in Plants. Molecular Genetics, Cell Biology and Physiology. Cabi Publishing, UK, pp. 203-218.

Malluta, E.F., Decker, P., Stambuk, B.U., 2000. The Kluyver effect for trehalose in Saccharomyces cerevisiae. J. Basic Microbiol. 40, 199-205.

Martínez-Monzó, J., Martínez-Navarrete, N., Chiralt, A., Fito, P., 1998. Mechanical and structural changes in apple (var. Granny smith) due to vacuum impregnation with cryoprotectants. J. Food Sci. 63, 499503.

Mavroudis, N., 2003. Mass transport in apple tissue - effects on tissue structure and osmotic processing conditions. PhD Thesis, Food Engineering Department, Lund University, Sweden.

Phillips, K.M., Wunderlich, K.M., Holden, J.M., Exler, J., Gebhardt, S.E., Haytowitz, D.B., Beecher, G.R., Doherty, R.F., 2005. Stability of 5 -methyltetrahydrofolate in frozen fresh fruits and vegetables. Food Chem. 92, 587-595.

Sabri, N., Pelissier, B., Teissié, J., 1996. Electropermeabilization of intact maize cells induces an oxidative stress. Euro. J. Biochem. 238, 737-743.

Smallwood, M., Bowles, D.J., 2002. Plants in a cold climate. Philos. Trans. Roy. Soc. London. Ser. B: Biol. Sci. 357, 831-847.

Somersalo, S., Krause, G.H., 1990. Effects of freezing and subsequent light stress on photosynthesis of spinach leaves. Plant Physiol. Biochem. 28, 467-475.

Stambuk, B.U., Araujo, P.S., Panek, A.D., Serrano, R., 1996. Kinetics and energetics of trehalose transport in Saccharomyces cerevisiae. Euro. J. Biochem. 237, 876-881.

Stanley, D.W., 1991. Biological membrane deterioration and associated quality losses in food tissues. Crit. Rev. Food Sci. Nutrit. 30, 487-553.

Taiwo, K., Eshtiaghi, M.N., Ade-Omowaye, B.I.O., Knorr, D., 2003. Osmotic dehydration of strawberry halves: influence of osmotic agents and pre-treatment methods on mass transfer and product characteristics. Int. J. Food Sci. Technol. 38, 693-707.

Taiz, L., Zeiger, E., 2002. Plant Physiology. Sinauer Associates, Inc.

Turan, M., Ozgul, M., Kocaman, A., 2007. Freezing tolerance affected by mineral application during cold-acclimated conditions in some cool crop seedlings. Commun. Soil Sci. Plant Anal. 38, 1047-1060.

Yancey, P.H., 2005. Organic osmolytes as compatible, metabolic and counteracting cytoprotectants in high osmolarity and other stresses. J. Exp. Biol. 208, 2819-2830. 